\title{
El discurso de Anténor en la llíada
}

\author{
LUCIA ADRIANA LIÑARES \\ Universidad de Buenos Aires - COMICET
}

\begin{abstract}
RESUMO: O presente trabalho considera que o discurso na relação comunicativa, reproduz as atitudes e valores compartilhados por um grupo, ao qual definem no marco social. O discurso de Antenor na llíada oferece características específicas que o distinguem dos outros anciãos locutores: à diferença destes, que manifestam modelos "individuais", o discurso de Antenor representa cada um dos velhos conselheiros de Tróia, e em vista disto, simboliza uma personalidade "social".
\end{abstract}

PALAVRAS-CHAVE: Discurso, comunicação, carácter individual, carácter social, anciãos, lliada, texto, contexto.

El presente trabajo forma parte de un proyecto de investigación ${ }^{1}$ sobre los viejos y la vejez en la Ilíad ${ }^{2}$. Dicha investigación, desarrollada metodológicamente en forma interdisciplinaria a partir de técnicas de análisis del discurso ${ }^{3}$, interpreta a la epopeya homérica como una obra literaria en la que los personajes se caracterizan por explicitar, a través de sus intervenciones, una personalidad "diferenciada", en el marco de un "repertorio tradicional"4. Así como el habla individual "simboliza"la personalidad que representa, el discurso en el ejercicio comunicativo permite reproducir aquellas actitudes $\mathrm{Y}$ valores compartidos por un grupo que definen a ese grupo en el marco de su categoria social ${ }^{5}$. Tanto el discurso como la comunicación que se estabelece a través del discurso permiten definir las distintas categorias sociales. Los individuos se prestentan también como miembros de grupos y participan de una estructura social que (inter)actúa por medio del discurso ${ }^{6}$. Los ancianos en la Ilíada no sólo se jerarqizan como individuos, sino también como miembros de un grupo; expresan Y comunican a los no viejos actitudes enmarcadas en la identificación de su categoria. Este conjunto de actitudes, valores y ceencias compartidos por los viejos se puede definir ${ }^{7}$ por medio de su reconstrucción a partir del discurso (como única fuente de información) y de su reproducción en situaciones comunicativas ${ }^{8}$. El habla personal es un medio por el cual el individuo desarrolla y define su personalidad frente a los demás ${ }^{9}$. El discurso tiene también funciones comunicativas y sociales, ya que permite la difusion y reprodución de creencias y opiniones sociales.

El punto de partida para el análisis del proyeto global ha consistido en responder a dos preguntas: qué se comunica y cómo se comunica ${ }^{10}$. La primera cuestión tiene que ver con la información proporcionada por el discurso de los viejos, la cual se refiere tanto a la expresión de variables individuales como también a aquellas actitudes y creencias (sociales) compartidas por el grupo. La segunda cuestión (cómo se comunica) da cuenta de mecanismos de reconstrucción. Las estructuras mentales subyacentes de los viejos como individuos durante la acción comunicativa, se han reconstruído de un modo más o menos abstracto a partir de la interpretación de los actos de habla expresados: vale decir que se ha obtenido información directa sobre el conocimiento, las creencias (como base de datos), los deseos y necesidades (como motivación para la acción) 
y las intenciones y propósitos (como planos de acción) de cada uno de ellos ${ }^{11}$. Por otra parte, el modo en que los viejos forman y tranmiten creencias y opiniones grupales se refiere más bien al proceso mismo de producción discursiva. No se ha intentado realizar la reproducción de este proceso; simplesmente se consideraron las evidencias individuales, y se las interpretó de acuerdo con operaciones de abstracción, descontextualización y generalización $n^{12}$. Las creencias y opiniones grupales fueron vistas como un resultado del proceso, no al nivel puramente individual, sino más bien como una propriedad de los miembros de un grupo ${ }^{13}$. El corpus abarcó la totalidad de las intervenciones directas (por meio del discurso) e indirectas (por referencia) de los ancianos en la Ilíada (Néstor, Fénix, Príamo, Crises, Calcas y Anténor), y además las referencias generales sobre los ancianos y vejez, las cuales han permitido corroborar la existencia de los valores y actitudes sociales que los ancianos de la realidad iliádica reproducen a través de su discurso (los ancianos no locutores Peleo, Menetio, Tideo y Neleo, especialmente el primero, funcionan como puntos de referencia para interpretar dichos valores): la importancia de la educación, el dolor por la vejez y la pérdida del hijo, el valor heroico en la juventud pasada, la elocuencia del hombre mayor y el respeto que se les confiere en virtud de sus funciones sociales (vate, sacerdote, consejero, rey, etc.).

El presente trabajo se ocupa especificamente del discurso de Anténor en la Ilíada, el cual evidencia una característica particular que lo distingue de los demás viejos locutores: éstos, como ya se ha señalado, son considerados hablantes "diferenciados", que comparten un conjunto de actitudes y valores los cuales reproducen por medio del discurso y la interacción comunicativa. Anténor por su parte no es un hablante "diferenciado"; representa más bien a cada uno de los ancianos en Troya. No se yergue como individuo para proyectar, por médio de su discurso, un modelo particular de la vejez, sino que, en todo caso, su discurso somboliza una personalidad "social". La organización interna de su discurso y las funciones que lo definen respecto de las necesidades dramáticas de la obra, se vinculan estrechamente con los rasgos fundamentales que caracterizan a los viejos troyanos. Estos consejeros son presentados no como individuos, sino como un grupo; inclusive el discurso emitido por ellos muestra con una sola voz enunciadora (aunque la narración define esta enunciación como una "conversación entre unos y otros": (Cf. III, 155). De todo el conjunto, sólo Anténor interviene en la interacción. Su discurso permite la "reconstrucción" del comportamiento discursivo de los viejos en Troya. Este proceso de reconstrucción menifiesta una dirección inversa al utilizado para interpretar el resto de los discursos de los viejos: en los demás casos, se consideraban las evidencias individuales, y se las interpretaba de acuerdo con operaciones de abstracción y generalización, de modo de "reconstruir" el sistema de valores identificativo de la categoria social de la viejez ${ }^{14}$. En el caso de Anténor, su discurso representa la voz generalizada y abstracta de los viejos troyanos, a partir de la cual puede "recuperarse" cada instancia individual".

El grupo de viejos troyanos es definido ${ }^{16}$ en virtud de tres rasgos fundamentales (Cf. III, 146 sq.):

1) su presencia junto al rey Príamo ${ }^{17}$ :

Hoi dámphi Príamon (...)

hérato demogérontes epì Skaiêisi pýleisi, ... (III, 146-149)

2) su no participación en el combate (debito a la edad avanzada):

... géraï dè polémoio pepauménoi, ... (III, 150)

3) su capacidad como arengadores (ilustrada por medio de un símil):

... all'agorétai

esthlói, tettigessin eoikótes, hoi te kath'hýlen

dendréoi ephezómenoi ópa leirióessan hieîsi;... (III, 150-152) 
El discurso que los presenta con una sola voz enunciadora (III, 156-160) define su posición (global) respecto de Helena. La justifican por su belleza:

ou némesis Tróas kaì eüknémidas Akhauoùs

toiêid'amphi gynaiki polýn khrónon álgea páskhein;... (III, 156-157)

Ilustran esta justificación con un simil que vincula a Helena con las divindades:

... ainôs athanátheisi theêis eis ôpa éoiken; ... (III, 158)

En la segunda parte del discurso se expoen sus verdaderas preferencias, articuladas por medio de una expresión desiderativa:

... allá kai hôs toíe per eô̂.'en neusì neéstho.

med'hemín tekéessi t'opísso pêma lípoito. (III, 159-160)

Vale decir que los ancianos troyanos son definidos de acuerdo con tres rasgos caracterizadores (elocuencia, presencia junto al rey, no participación en el combate) y en la expresión conjunta de su posición frente al enemigo (reconocimiento elogioso y explicitación de deseos y preferencias).

Este escenario general en las murallas de Troya enmarca la primera alocución de Príamo en interacción con Helena (III, 161 sq. $)^{18}$. La primera intervención de Anténor (III, 202-224) especifica a Odisseo como referente proporcionado por Helena en respuesta a Príamo. El rey ha solicitado información sobre Odiseo, al que describe visualmente sin identificarlo (Cf. III, 192-198). Helená (Cf. III, 200-202) define al héroe de acuerdo con su rasgo esencial de elocuencia y habilidad discursiva:

... eidòs pantoíous te dólous kaì médea pykná. (III, 202)

Anténor ratifica la descripción dada por Helena, articulando el elogio a Odiseo por medio de una reminiscencia ${ }^{19}$ (recuerda cuando Odiseo fue a Troya con Menelao en calidad de embajador para recuperar a Helena, y él los agasajó en su palacio). Este discurso ${ }^{20}$ jerarquiza su capacidad de elocuencia en el recuerdo elogioso de Odiseo; la reminiscencia realza también la habilidad de Anténor para proyectar su descripción del héroe en una imagen que reúne, por un lado, los aspectos visuales percibidos por Príamo desde la muralla (vale decir, la apariencia de Odiseo), y por otro, la capacidad mayor del Laertíada: su elocuencia. Esta imagen se encuadra en una situación del pasado, y se jerarquiza en virtud de una triple relación comparativa: Odisseo frente a Menela (en cuanto a apariencia y habilidad en el consejo ${ }^{21}$, el aspecto visual frente a la capacidad oral (Cf. III, 219) y el símil de los copos de nieve (III, 222) para ilustrar el despliegue de la palabra pronunciada.

Más adelante, en los versos 259 sq., la intervención (no discursiva) de Anténor define otro rasgo caracterizador: su presencia junto ao rey. Cuando Príamo se dirige al campo de batalla para ratificar los juramentos frente al enemigo (y también cuando regresa a Troya), Anténor se ubica en el carro a su lado:

\section{... pàr dé hoi Anténor perikalléa béseto díphron; ... (III, 262 y 312)}

Su siguinte (y última) intervención se produce en el libro VII. Nuevamente los troyanos se reúnen en el ágora junto al palacio de Príamo (VII, 345 sq.). Anténor es el primero en hablar (VII, 348-353). El pedido de que lo escuchen (VII, 348-349) prepara el acto de habla principal ${ }^{22}$ : exhorta a devolver a Helena:

\section{... deût'áget', Argíen Helénen kaì ktémath' hám' autéi dóomen Atreídeisin ágein; ... (VII, 350-351)}

Justifica la propuesta con una declaración descriptiva sobre la situación presente: 


\author{
... nûn d'hórkia pistà \\ pseusámenoi makhómestha; ... (VII, 351-352)
}

Proporciona una evaluación — negativa — de la misma, debido a que explicita sus expectativas (negadas):

\author{
... tô oú nú ti kérdion hémin \\ élpimai ekteléesthai, hína mè réxomen hôde. (VII, 352-353)
}

Este discurso jerarquiza, por un lado, a Anténor como primer y único anciano consejero. Además, la propuesta define con nitidez su posición respecto del enemigo, posición que se ve doblemente justificada: por medio de la evaluación de la situación, y en virtud de la especificación y alcance de sus temores declarados. Este parlamento provoca la intervención de Paris (VII, 357 sq.) quien no pone en duda la capacidad de elocuencia de Anténor, sino que manifesta su desagrado respecto de su propuesta. Acto seguido realiza él mismo la declaración de sus propósitos (no devolver a Helena, aunque sí los demás bienes - Cf. 362 sq. - ). La última palabra en el ágora la tiene Príamo (VII, 368 sq.); su intervención preanuncia la tregua para honrar a los muertos (VII, 412 sq.).

En síntesis: el discurso y las interenciones de Anténor proporcionan información sobre los siguientes aspectos:

1. elocuencia del anciano consejero, que se manifesta discursivamente en dos formas, de acuerdo con la posición asumida frente al enemigo:

1.1. reconocimiento elogioso (imagm - anmarcada en un recuerdo pasado - compuesta a partir de elementos de carácter visual y auditivo);

1.2. exhortación (solicitud de que lo escuschen + propuesta concreta respecto de Helena);

2. presencia junto ao rey en el consejo y en situaciones pacificas - ratificación de juramentos -, no en el combate.

Si se vincula este cuadro descriptivo con los rasgos que define a los consejeros troyanos como grupo, se hace evidente que Anténor ilustra en su persona la capacidad discursiva de todos; además su conducta (verbal y no verbal) responde a la configuración general. $Y$, lo que es más importante, la enunciación de sus intenciones y propósitos (vale decir, la propuesta de devolver a Helena) traduce en un acto exhortativo los deseos y preferencias del grupo de ancianos, sugeridos en el discurso global (en la expresión desiderativa). Los deseos y preferencias funcionan como motivación para la acción ${ }^{23}$; estas preferencias son explicitadas por los viejos como grupo; y el discurso particular de Anténor las hace funcionar en un verdadero acto de habla exhortativo.

Un detalle léxico ( además de la reiteración del verbo 'agoreuo' en todas las escenas mencionadas) ilustra en forma cabal esta particular capacidad de reconstrucción discursiva "social": el nombre mismo de Anténor (antí + anér) da cuenta, en una de sus posibilidades significativas $^{24}$, de la función principal de este anciano: su voz es la voz de todos; su esencia es estar "en lugar de" los demás hombres de su clase.

\title{
Notas
}

1- Desarrollado desde 1986 durante las becas otorgadas por el Consejo Nacional de Investigaciones Científicas y Técnicas: Iniciación (Organización y funciones del discurso de Néstor en la Ilíada); Perfeccionamiento (Las Modalidades del discurso en la Iliada: Néstor, Fénix y Príamo), y Prórroga de Perfeccionamento (Modelos de comunicación en la llíada: la concepción de la vejez y el análisis del discurso), bajo la dirección de la Lic. Elena F. Hüber 
(Univ. Buenos Aires).

2 - Para el presente trabajo, se ha utilizado la siguiente edición: Monro \& Allen, $1920^{3}$. Las normas seguidas para la transliteración de palavras en griego son proporcionadas por Ramón Alcalde y Eduardo J. Prieto, directores de la Biblioteca de Cultura Clásica Paidós, en Kirk, 1968, 1 ed. 1962, p. 347-349: se siguen allí las reglas de la lengua española para la acentuación y la puntuación.

3 - Los conceptos se apoyan principalmente en las propuestas teóricas de Teun van Dijk en las notas siguientes se proporcionarán algunas referencias pertinentes al presente trabajo).

4 - El artículo de Paul Friedrich \& James Redfield (Friedrich \& Redfield, 1978, p. 253-288) se constituye en punto de partida para esta interpretación de los personajes homéricos como hablantes, identificados por "idiolectos" (sin perder de vista el sustrato común que se define como "repertorio tradicional"). Cf. también la polémica a partir de este artículo (Messing, 1981, p. 888-900 y Friedrich \& Redfield, 1981, p. 901-903).

5 - Se ha tenido presente el enfoque metodológico de Teun van Dijk para el estudio del racismo y su vinculación con el discurso; las obras consultadas de este autor dan cuenta de una evolución en el estudio teórico del discurso: a partir de propuestas de carácter teóriçọ, se procede a la aplicación prática en el análisis del discurso y el racismo en la sociedad occidental. El fundamento que subyace a su investigación jerarquiza el papel del discurso en la reprodución de actitudes sociales. Esta noción que subraya la función social de la comunicación se constituye en el punto de partida para el presente trabajo: así como se tuvieron en cuenta las pautas teóricas fundamentales de van Dijk (van Dijk, 1984, 1 ed. 1977) en la etapas anteriores de investigación, en esta oportunidad la metodologia de van Dijk orienta y estimula la elaboración de un método de análisis proprio, cuya premisa fundamental consiste en procurar su adecuasión y utilidad respecto del estudio del discurso homérico.

6 - Cf. van Dijk (van Dijk, 1988, p. 131-180): en el marco interdisciplinario que estudia la (re)producción del racismo, se contemplan tres dimensiones de análisis: discursiva, interaccional y cognitiva: la primera perspectiva da cuenta del contenido y de las estrategias discursivas, la segunda se interesa en las funciones sociales del discurso como una forma de (inter) acción baseada en un grupo (los individuos están considerados como miembros de un grupo y participan de una estructura sociopolítica); la perspectiva cognitiva permite analizar las estructuras y las estrategias del prejuicio étnico y del procesamiento de la información social en general. A partir de este planteo, la presente investigación se ocupa de los parlamentos de los viejos en la Ilíada, tiendo en cuenta las tres dimensiones de análisis: el discurso individual" de cada viejo; las interacciones comunicativas de los viejos como grupo; y el discurso en tanto modo de expresión y reproducción de actitudes y normas (sociales) formadas, adaptadas y compartidas.

7 - Se ha presentado la siguiente ponencia en el marco de las VI Jornadas de Estudios Clásicos (Universidad Católica Argentina, Facultad de Filosofía y Letras, Buenos Aires, 27 de junio de 1991): "Los viejos en la Ilíada: comunicación y modelos argumentativos".

8 - Van Dijk estudia (van Dijk, 1984) el papel reprodutor del racismo en la conversación cotidiana; este trabajo ha proporcionado las pautas generales para el análisis discursivo, en tanto que van Dijk, 1988, aporta el marco conceptual y metodológico del presente artículo.

9 - Este principio fundamenta el análisis de Friedrich \& Redfield (Friedrich \& Redfield, 1978, p. 264) sobre el "habla" de Aquiles.

10 - La teoría general de la comunicación (desde Aristóteles hasta los semióticos modernos) se basa sobre un modelo único, el modelo de código (la comunicación se logra codificando y decodificando mesnajes); el modelo inferencial propuesto recientemente define a la comunicación según el proceso de producir e interpretar evidencia. Para el planteo y desarrollo 
de estas dos preguntas fundamentales en el estudio de la comunicación (qué y cómo se comunica), Cf. a D. Sperber \& D. Wilson (Sperber \& Wilson, 1986, cap. 1).

11 - Esta parte de la investigación se ha desarrollado durante la beca de Perfeccionamiento (Cf. supra n. 1).

12 - El proceso de producción del discurso es analizado y reconstruido por van Dijk (van Dijk, 1984, p. 24 sq. Cf. la gráfica del procesamiento cognitivo de la información en p. 27, y también en p. 46-48). Las operaciones de abstracción, descontextualización y generalización que se adjudican al sujeto mismo que produce un discurso (en la comunicación verbal humana), serán utilizadas en el presente análisis de los personajes homéricos como un mecanismo de interpretación para acceder, a partir de los modelos individuales explicitados en los discursos, al conjunto de valores subyacente. Vle decir que no se adjudica a los personajes la capasidad de abstracción: simplesmente se subrayan las evidencias individuales, y de ellas se infieren los elementos en común.

13 - Cf. van Dijk (van Dijk, 1984, p. 3) en cuanto al planteo del processo de la información social; para los personajes homéricos, el resultado del proceso se define e interpreta exclusivamente a partir del discurso, como su capacidad fundamental de reproducir opiniones y actitudes globales.

14 - Vale decir que, en virtud de las pautas teóricas utilizadas para decribir la comunicación verbal humana y reconstruirla como un procesamiento de información, en el presente trabajo se interpretan los discursos como el resultado de un proceso dinámico que solamente se hace evidente en su explicitación individual. Las etapas del procesamiento de producción discursiva se traducen y definen (en esta investigación) en etapas de interpretación y análisis; los discursos proporcionan las pautas individuales, a partir de las cuales se recupera la información percebida y se reconstruyen los valores grupales. Ante la imposibilidad de "restaurar" la mente homérica. La cualidad dinamica se traslada al proceso mismo de interpretación.

15 - En el procesamiento de producción del discurso, van Dijk reconoce como última fase la recuperación y (re)producción de información (estos pasos del procesamiento cognoscitivo del discurso son resumidos en van Dijk, 1983, 1 ed. 1978, p. 77-113); en la presente investigación, los mecanismos de "recuperación" y "reconstrucción" son inherentes a la interpretación de los modelos individuales: vale decir que definen y explicitan una etapa de análisis.

16 - El escenario de Troya como situación (global) de enunciación se presenta en el marco del catálogo de héroes (II, 786 sq.).

17 - El análisis de sus discursos jerarquiza la individualidad de su carácter (Cf. infra n. 19).

18 - El estudio de Príamo a través del análisis lingüístico de su discurso en la Ilíada ejemplifica en dos trabajos realizados el método que define el perfil global de la investigación: la reconstrucción de las relaciones comunicativas entre los personajes hablantes, que reproducen las actitudes proposicionales de cada uno. Se ha participado de dos reuniones científicas: en la V Reunião Anual da Sociedade Brasileira de Estudos Clássicos, realizada en el período del 23 al 27 de julio de 1990 en Garibaldi, RS (Brasil), se ha presentado el trabajo: "El discurso de Príamo en la Ilíada: la referencia". En el XI Simposio Nacional de Estudios Clásicos, desarrollado en Rosario, Santa Fe (Argentina) los días 17 al 22 de sptiembre de 1990, se ha participado con la ponencia: "El discurso de Príamo en la Ilíada: mecanismos de organización".

19 - Para algunas consideraciones sonre la estructura del parlamento cf. Julia H. Gaisser (Gaisser, 1969, p. 1-43, especialmente p. 6). 
20 - III, 204-224:

Tèn d'aût'Anténor pepnüménos antion éuda: 'ô gýnai, ê mália toûto épos nèmertès éeipes; éde gàr deûro pot'élythe díos Odysséus seû hének'aggelíes sýn areïphiloi Menelaoi; toùs d'egò exéinissa kaì en megároisi phílesa, amphotéron dè phyèn edáen kaì médea pykná all'hóte dè Tróessin en agroménoisin émikhthen, stánton mèn Menélaos hypéirekhen euréas ómous, ámpho d'hezoméno geraróteros êen Odysséus; all'hóte dè mýthous kaì médea pâsin hýphainon, étoi mèn Menélaos epitrokháden agóreue, paûra mén, allá mála ligéos, epei ou polýmuthos oud'aphamartoepés; $\hat{e}$ kaì génei hýsteros êen. all'hóte dè polýmetis anaíxeien Odysséus, atásken, hypaì dè ídeske katá khthonós ómmata péxas, skêptron d'out'opíso oúte proprenès enóma, all'astemphés ékhesken, aídrei photì eoikós; faíes ke zákotón té tin'émmenai áphrona t'aútos, all'hóte dè ópa te megálen ek stétheos heíe kaì épea niphádessin eoikóta kheimeríeisin, ouk àn épeit'Odyseí g'erísseie bròtos állos; ou tóte g'hód'Odyséos agassámeth'eîdos idóntes.

21 - Estas referencias constituyen uno de los puntos de partida para reconocer en Homero la creación de estilos retóricos; cf. los alcances de la polémica en torno de este tema en Gorge A. Kennedy (Kennedy, 1957, p. 23-25).

22 - Cf. van Dijk (van Dijk, 1984, 1 ed. 1977, p. 302 sq.) para el enálisis de las secuencias de actos de habla.

23 - Cf. van Dijk (van Dijk, 1984, 1 ed. 1977, p. 256 sq.) para la descripción de las estructuras mentales (supuestas) de acción.

24 - Cf. Pierre Chantraine (Chantraine, 1968), artículo "anta, anten, anti", sección 2.

\section{Referencias Bibliográficas}

CHANTRAINE, Pierre, Dictionnaire Étymologique de la Langue Grecque. Paris: Klincksieck, 1953.

VAN DIJK, Teun A., Texto y contexto. Traducción de Juan Domingo Moyano. Madrid: Cátedra, 1984 (1 ed., 1977).

VAN DIJK, Teun A., Estructuras y funciones del discurso. México: Siglo XXI, 1983. (Serie de conferencias pronunciadas en el Departamento de Lingüística de la Facultad de Humanidades de la Univ. de Puerto Rico en 1978).

VAN DIJK, Teun A., Prejudice in discourse. Amsterdam: Benjamins, 1984.

VAN DIJK, Teun A., El discurso y la reproducción del racismo. Lenguaje en contexto. Buenos Aires, v. 1, n. 1-2, p. 313-180, 1988. 
FRIEDRICH, Paul \& REDFIELD James, Speech as a personality symbol: The case of Achilles. Language, Baltimore, vol. 54, n. 2, p. 253-288, 1948.

FRIEDRICH, Paul, Contra Messing. Language: Baltimore, vol. 57, n. 4, p. 901-903, 1981.

GAISSER, Julia H. A structural analysis of the digressions in the Iliad and the Odyssey. Harvard Studies in Classical Philosogy, Cambridge, Mass., v. 73, p. 1-43, 1969.

KENNEDY, George A. The ancient dispute over Rhetoric in Homer. American Journal of Philology. Baltimore, v. 78, n. 1, p. 23-35, 1957.

KIRK, Geoffrey S., Los poemas de Homero. Traducción de Eduardo J. Prieto \& Ramón Alcalde. Buenos Aires: Paidós, 1968 (1. ed., 1962).

MESSING, Gordon, On weighing Achilles 'winged words. Language; Baltimore, vol. 57, n. 4, p. 888-900, 1981.

MONRO, David \& ALLEN, Thomas W., Homeri Opera. $3^{\mathrm{a}}$ ed., Oxford: Clarendon Press, 1920 , vols. 1 e 2 ..

SPERBER, D. \& WILSON, D. Relevance, communication and cognition. Cambridge Mass.: Harvard Univ. Press, 1968.

LIÑARES, L. A. Antenor's speech in the Iliad. Classica, São Paulo, 7/8: 173-180, 1994/1995.

\begin{abstract}
This paper argues that the discourse, in communicative situations, reproduces attitudes and values shared by a group, which is defines in the social structure. The speech of Antenor in the lliad has specific traits which distinguish him from the other old speakers: while these present "individual" models, Antenor's speach represents every eldery Troyan counsellor and thus symbolizes a "social" personality.
\end{abstract}

KEY WORDS: Discourse, communication, individual personality, social personality, eldery speakers, Iliad, text, context. 